\title{
Rechtsgeschichte
}

\section{Achim Landwehr}

Schmach und Schande 
non conta più tanto per la dignità della sua disciplina ma per la capacità di guadagnarsi la fiducia del potere politico? Mi sembra che si dimostri che dobbiamo uscire dal generico: un campo così vasto richiede delle contestualizzazioni. Ad esempio, di quale Italia si parla? Delle città soltanto ora inglobate nello Stato pontificio, o di città che sono nel pieno dello splendore culturale (non solo Firenze), o di ordinamenti in cui i giuristi si avviano ora - come avviene specie a Napoli - ad avere un ruolo enorme di mediazione politico-sociale? Quel Forteguerri (che neppure è autore sicuro dell'opera attribuitagli) opera con Alfonso il Magnanimo come il Valla: non vorrà dire niente? E che il Signorolo e il Garati fossero di ambiente lombardo? Dalle idee ai contesti: a questo punto è necessario se si vuole andare avanti su questo grande problema.

Non continuo, se non per mettere in guardia su dettagli. Non mi sembra ad esempio che il periodo degli Sforza sia particolarmente da studiare (tra gli altri si v. Martines, la Villata Di Renzo, Massetto, la Leverotti), e lo stesso vale per la communis opinio, oggetto anni fa d'un libro fondamentale di Lombardi Vallauri. Aggiungerei, dato che Gilli chiede giustamente dati ssociali<, che c'era il grande libro di Marino Berengo sulla città europea, una vera miniera, e sui canonisti e teologi del '300-'400 come tra-

\section{Schmach und Schande*}

Man ist geneigt, vor dieser Arbeit bereits nach wenigen Seiten den Hut zu ziehen. Denn recht schnell zeichnet sich ab, dass Matthias Lentz mit seiner Dissertation ein beispielhaftes Stück Sozialgeschichte des Rechts vorgelegt hat, lasciare l'opera sulla giustizia di Paolo Prodi? Fa anche una certa impressione vedere ben poco utilizzati Caprioli e Troje, e per nulla Palazzini Finetti, Abbondanza, Osler ...

Per finire, cautela nell'accostarsi ai passi latini delle note trascritti da edizioni antiche, ricchi di errori (come dire?) più frequenti del normale; errori sono frequenti anche nei nomi degli autori delle opere ricordate (leggiamo di D. Segolini, U. Niccolini, G. Minucci, C. Storti Stocchi, Lucca da Penne ecc., per non dire delle varianti di Baumgärtner). I due ordini di errori fanno pensare a un'opera chiusa di corsa. Perciò il Caccialupi diviene canonista, Accursio è ricordato in Dante invece di Francesco (ma c'è il libro di Kay!), Ugo Benzi diviene fiorentino, Giovanni d'Andrea il primo professore laico di diritto canonico (diabolica probatio!); c'è anche un >Diplovatazzi Tommaso<.

L'idea era buona ed è stata portata avanti assai bene fino a un certo punto. Alla fine ha sopraffatto la massa della ricerca già effettuata, entro la quale si sono potuti seguire solo alcuni filoni. Peccato, il paradiso già dei giuristi è rimasto lontano per il ricercatore di oggi: forse, stavolta sì, c'era bisogno di un lavoro collettivo, di una ricerca a più voci e competenze.

Mario Ascheri

das in mehrfacher Hinsicht zu überzeugen weiß. Die Arbeit ist sprachlich gelungen und gut lesbar, glänzt mit einer stringenten Argumentation und beeindruckt vor allem durch die Auffächerung eines Quellenkorpus, das bisher nur sporadisch

\footnotetext{
* Matthias Lentz, Konflikt, Ehre, Ordnung. Untersuchungen zu den Schmähbriefen und Schandbildern des späten Mittelalters und der frühen Neuzeit (ca. I350 bis I600). Mit einem illustrierten Katalog der Überlieferung, Hannover: Hahnsche Buchhandlung 2004, 383 S., ISBN 3-7752-60I7-X
} 
und zumeist illustrierend in den Geschichtswissenschaften Berücksichtigung fand. Doch im weiteren Verlauf der Lektüre sieht man sich zumindest zeitweise gezwungen, die Hand wieder von der Hutkrempe zu nehmen und sich das Kompliment nochmals genauer durch den Kopf gehen zu lassen.

Doch bevor wir auf dieses Problem näher zu sprechen kommen, gilt es zunächst, den Gegenstand kurz zu erläutern. Lentz hat sich der spätmittelalterlichen und frühneuzeitlichen Gattung der Schmähbriefe und Schandbilder angenommen. Dabei handelt es sich um Quellen mit TextBild-Kombinationen, die zumeist dann zum Einsatz kamen, wenn ein Schuldner oder seine Bürgen gegenüber einem Gläubiger ihren Pflichten nicht nachkamen. Erhielt ein Gläubiger auch nach mehrfachen Versuchen beispielsweise das von ihm vergebene Darlehen nicht zurück, konnte er zum Mittel der Schmähbriefe und Schandbilder greifen. Darin wurde sowohl bildlich als auch textlich in zumeist recht derber Weise das Vergehen der Beschuldigten dargestellt und an öffentlichen Plätzen bekannt gemacht, um sie auf diese Weise an einer empfindlichen Stelle zu treffen: an ihrer Ehre, die innerhalb vormoderner Gesellschaften wesentlich über die soziale Stellung entschied. Dass sich die Gläubiger nicht anders zu helfen wussten, wird durch die Deutlichkeit der Darstellungen unterstrichen. Die Schandbilder zeigen häufig Schuldner und Bürgen rücklings auf Schweinen oder Eseln sitzend, gevierteilt oder gehängt, und ihre Siegel, mit denen sie die nicht eingehaltene Schuldverschreibung beglaubigt hatten, mit Kot beschmutzt. Stand den Gläubigern lange Zeit kein anderer Weg offen, als auf diese Weise ihren Forderungen Nachdruck zu verleihen, war es nur konsequent, dass Schmähbriefe und Schandbilder im späten I6. Jahrhundert allmählich verschwanden, als entsprechende Streitigkeiten zunehmend vor Gericht ausgetragen wurden.

Aufgrund der umfassenden Erarbeitung dieser Quellenbasis kann Lentz für die weitere Einordnung der Schmähbriefe und Schandbilder einige wichtige Ergebnisse zutage fördern. So erteilt er der möglicherweise sich einstellenden

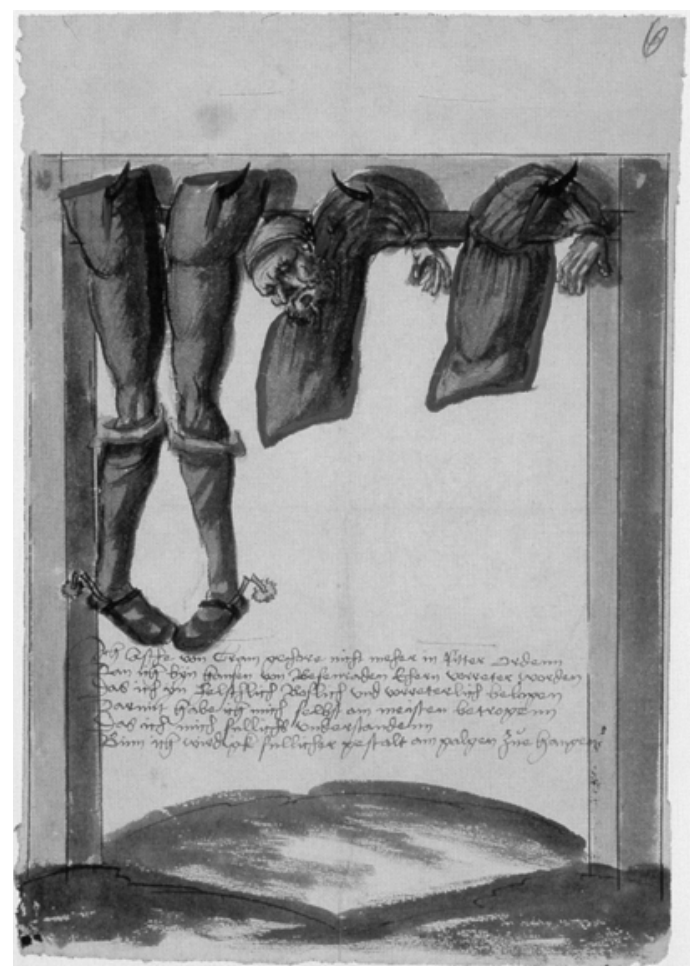

Einschätzung eine Absage, bei diesen Dokumenten handele es sich um Belege für Konflikte, die sich vornehmlich zwischen Obrigkeit und Untertanen abspielten. Dem war offensichtlich nicht so. Schmähbriefe und Schandbilder können nicht als Manifestation widerständigen Eigen- 
sinns von Machtunterworfenen gedeutet werden, sondern finden sich ausschließlich im Kontext vertraglicher Schuldvereinbarungen, deren Konditionen vermeintlich oder tatsächlich nicht eingehalten wurden. Als grundlegend schätzt Lentz die Untersuchung dieser Quellen richtigerweise deswegen ein, weil sie nicht nur Einblicke in spätmittelalterliche und frühneuzeitliche Rechtsverständnisse gewähren, sondern darüber hinaus auch soziale Grundlagen dieses Zeitraums freilegen. Ehre, ein gegebenes Wort, persönliche Bindungen und ungeschriebene Regeln spielten zur Absicherung gegenseitiger Erwartungen eine entscheidende Rolle - weshalb man auch zu bildlich und sprachlich drastischen Mitteln greifen musste, wenn eine solche Erwartungssicherung enttäuscht wurde. Darüber hinaus zeigt Lentz aber auch, welche Rolle entgegen verbreiteter begrifflicher Zurückhaltungen für vormoderne Verhältnisse - die Öffentlichkeit für dieses Medium spielte. Denn die Schmähbriefe und Schandbilder waren essentiell davon abhängig, »kundgetan « zu werden und mithin zumindest eine situative und lokale Öffentlichkeit herzustellen. Lentz unterstreicht daher auch zutreffend die Bedeutung eines in solcher Form variierten Öffentlichkeitsbegriffs für die Erforschung des Spätmittelalters und der Frühen Neuzeit.

Ein weiterer wichtiger Aspekt wird von Lentz leider nur gegen Ende seiner Untersuchung kurz angesprochen, hätte aber sicherlich eine insgesamt ausführlichere Würdigung verdient. Denn neben den Aspekten Öffentlichkeit und Ordnung wird als eine weitere Grundfrage vormoderner Rechtskultur die Verbindung von Schrift und Bild als Kennzeichen der untersuchten Quellengattung hervorgehoben. Wie Lentz richtig betont, wurde das Sehen als höchste Form der Erkenntnis bewertet, weshalb »offenbar-ma- chen « zumeist gleichbedeutend war mit »sichtbar-machen «. Gerade angesichts der in den vergangenen Jahren intensivierten Forschung zu mediengeschichtlichen Fragen hätte dieser Aspekt sicherlich mehr Aufmerksamkeit verdient als die wenigen Seiten, die Lentz ihm gegen Ende der Arbeit zubilligt. Nicht nur in der Geschichts-, sondern auch in der Literaturwissenschaft ist in den letzten Jahren - beispielsweise mit Blick auf Flugblätter - vielfach über Medien gearbeitet worden, die sich in ähnlicher Weise wie Schmähbriefe und Schandbilder durch eine Text-BildKombination auszeichnen.

Diese verpasste Chance, dem Untersuchungsmaterial weitere Erkenntnisse abzuringen, korrespondiert mit einem anderen Eindruck, der sich bei der Lektüre der Arbeit einstellt: Die Schmähbriefe und Schandbilder selbst werden in merkwürdiger Weise auf Distanz gehalten. Damit soll gesagt sein, dass die Quellen zumindest im ersten Teil der Arbeit kaum zum Gegenstand gemacht werden. Vielmehr konzentriert sich Lentz vor allem auf die rechtlichen und sozialen Rahmenbedingungen, führt der Leserschaft die Dokumente selbst aber nicht näher vor Augen. Diese Aufgabe überlässt er stattdessen gänzlich dem zweiten großen Teil der Arbeit, der einen umfangreichen, detaillierten, vorbildlich eingerichteten und reich bebilderten Katalog von exakt 200 Schmähbriefen und Schandbildern aus zahlreichen Archiven enthält. Eine stärkere Verschränkung der beiden Teile wäre wünschenswert gewesen.

Aber diesen Aspekt allzu sehr zu betonen, hieße eine eventuelle Schwäche gegenüber den vielen Stärken dieser Arbeit über Gebühr zu betonen. Denn allein die Tatsache, dass Lentz durch seine Arbeit der historischen Forschung erstmals den Schlüssel zu einer ganzen Quellengruppe geliefert hat, kann kaum hoch genug 
veranschlagt werden. Darüber hinaus wird der von ihm erstellte Katalog noch für lange Zeit wertvolle Dienste leisten. Führen wir daher als abschließende Bewertung in voller Überzeugung die Hand zur Kopfbedeckung und entbieten dem Autor ein aufrichtiges chapeau!

Achim Landwehr

\section{Gute Ordnung als Theorie und Praxis*}

Die Policey ist auf dem besten Wege, vom Aschenputtel zur Ikone der Frühneuzeitforschung zu werden. Manche Historiker sehen darin inzwischen ein Konzept, das geeignet ist, Grundprinzipien und Leitvorstellungen politischen Handelns in der Frühneuzeit zu charakterisieren. Peter Blickle etwa versteht Policey als Fundamentalprinzip, das »Sozialdisziplinierung und Absolutismus als Paradigmen zur Beschreibung der Frühen Neuzeit zunehmend verdrängt «. ${ }^{\mathbf{I}}$ Für diese Einschätzung spricht nicht nur die Quellennähe des Begriffs und die ungeheure Vielfalt der Lebensbereiche, die policeylicher Ordnung und Regulierung unterworfen waren, sondern auch die Tatsache, dass die Konkretheit und Ereignisbezogenheit policeylichen Handelns die Chance eröffnet, Staat und Herrschaft von den ihnen zugrunde liegenden Interaktionen und Praktiken her in den Blick zu nehmen. ${ }^{2}$ Die Tragfähigkeit des Policeykonzepts als Paradigma der Frühneuzeitforschung ist freilich jenseits des deutschen Sprachraums bislang noch kaum ausgelotet. ${ }^{3}$

Dies galt bislang auch für die französische police, die lediglich für das Paris des I8. Jahrhunderts einigermaßen gründlich untersucht war. Nun liegen zwei breiter angelegte Studien vor, die Möglichkeiten des Vergleichs eröffnen: die bei Peter Blickle entstandene Dissertation von Andrea Iseli zur bonne police und die auf eine juristische thèse zurückgehende Studie des an der Pariser École des Hautes Etudes en Sciences Sociales lehrenden Rechtshistorikers Paolo Napoli. Trotz zahlreicher Berührungspunkte sind die beiden Bücher grundverschieden. Iselis Arbeit verfolgt das Ziel, ein möglichst vollständiges Bild der frühneuzeitlichen police »in all ihren Facetten « nachzuzeichnen (I6) und dabei die Theorie ebenso einzubeziehen wie die beteiligten Institutionen, deren Kompetenzen und deren Praxis. Napoli verfolgt eine juristische (und im Kern politische) Fragestellung. Ihm geht es darum, die Grundlagen der spezifischen, vom juristischen Standpunkt aus hybriden, anomalen Rationalität der heutigen police herauszuarbeiten, indem er deren Anfänge seit dem Spätmittelalter und deren Wandel unter dem Einfluss der Aufklärung und der Französischen Revolution analysiert. Liest man beide Arbeiten, ergänzen sich ihre Ergebnisse zu einem dichten Bild.

Dies gilt vor allem für die Entwicklung des police-Konzepts und seine theoretische Durchdringung bis zum frühen I8. Jahrhundert, die mit unterschiedlicher Schwerpunktsetzung - in beiden Arbeiten behandelt wird. Napoli zufolge kristallisierte sich bereits im Frankreich des I3. Jahrhunderts ein erster konzeptioneller Kern dessen heraus, was später als police bezeichnet wurde: konkrete Regelungen und Maßnahmen zur Sicherstellung der Versorgung und der inne-

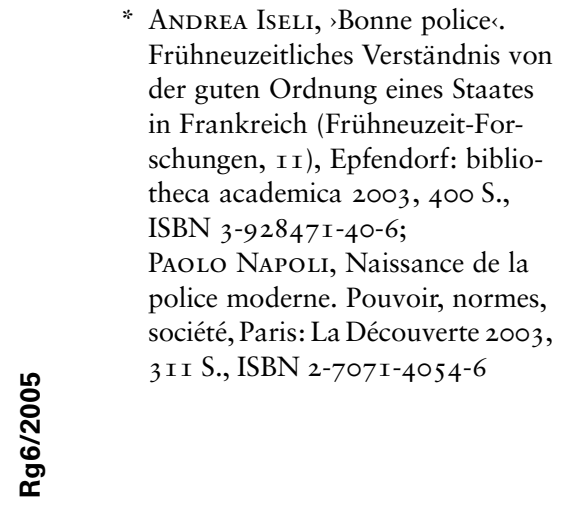

I Peter Blickle, Vorwort, in: Gute Policey als Politik im I6. Jahrhundert. Die Entstehung des öffentlichen Raumes in Oberdeutschland, hg. von DEMS. u. a., Frankfurt a. M. 2003, VII.

2 So jetzt mustergültig ANDrÉ Holenstein, >Gute Policey< und lokale Gesellschaft im Staat des ancien régime, 2 Bde., Epfendorf 2003 .
3 Einen ersten Überblick bietet bislang: Policey im Europa der Frühen Neuzeit, hg. von MichaEL STOLLEIS unter Mitarbeit von KARL HÄRTER und LOTHAR SCHILling, Frankfurt a. M. I996. 